\title{
Comportamiento a altas temperaturas de aceros inoxidables austeníticos refractarios: Formación de fase $\sigma$ y oxidación al aire
}

\author{
J. Botella $^{(*)}$, J. Almagro ${ }^{(*)}$, E. Otero ${ }^{(* *)}$, P. Hierro ${ }^{(* *)}$ y C. Merino ${ }^{(* *)}$
}

\begin{abstract}
Resumen Se oxidan al aire durante $2 \mathrm{~h}$ a $1.323 \mathrm{~K}$ tres aceros inoxidables: $25 \mathrm{Cr} 20 \mathrm{Ni}, 21 \mathrm{Cr} 11 \mathrm{Ni}_{(1)}$ y $21 \mathrm{Cr} 11 \mathrm{Ni}_{(2)}$. Los tres materiales forman capas de óxido de 1 a $3 \mu \mathrm{m}$ de espesor, con cristales externos de $\mathrm{MnCr}_{2} \mathrm{O}_{4}$ e internos de $\mathrm{Cr}_{2} \mathrm{O}_{3}$. Debajo del $\mathrm{Cr}_{2} \mathrm{O}_{3}$, en la intercara óxido -metal, se forma $\mathrm{SiO}_{2}$, en forma de precipitados intra e intergranulares en el metal, especialmente abundantes en los $21 \mathrm{Cr} 11 \mathrm{Ni}$. Las predicciones teóricas sobre las tendencias a la formación de fase se ven confirmadas tras un tratamiento de $400 \mathrm{~h}$ a $1.073 \mathrm{~K}$; el $25 \mathrm{Cr} 20 \mathrm{Ni}$ forma un $10 \%$ en volumen de $\sigma$, mientras que los aceros $21 \mathrm{Cr} 11 \mathrm{Ni}$ o no forman $\sigma$, o lo hacen escasamente.
\end{abstract}

Palabras clave: Aceros inoxidables austeníticos refractarios. Oxidación al aire. Altas temperaturas. Fase $\sigma$.

\section{High temperature behaviour of refractory austenitic stainless steels: $\sigma$-phase formation and oxidation in air}

\begin{abstract}
Three stainless steels: $25 \mathrm{Cr} 20 \mathrm{Ni}, 21 \mathrm{Cr} 11 \mathrm{Ni}_{(1)}$ and $21 \mathrm{Cr} 11 \mathrm{Ni}_{(2)}$ have been oxidised in air at $1,323 \mathrm{~K}$ for $2 \mathrm{~h}$. The three materials form oxide layers 1 to $3 \mu \mathrm{m}$ thick with $\mathrm{MnCr}_{2} \mathrm{O}_{4}$ external crystals and $\mathrm{Cr}_{2} \mathrm{O}_{3}$ inner ones. There are a $\mathrm{SiO}_{2}$ barrier below $\mathrm{Cr}_{2} \mathrm{O}_{3}$, in the oxide - metal interface. $\mathrm{SiO}_{2}$ also forms intra and intergranular precipitates in the metal, which are specially abundant in $21 \mathrm{Cr} 11 \mathrm{Ni}$ materials. The theoretical predictions about $\sigma$-phase formation tendencies are confirmed after a treatment at $1,073 \mathrm{~K}$ for $400 \mathrm{~h}$ : $25 \mathrm{Cr} 20 \mathrm{Ni}$ forms $10 \%$ vol. $\sigma$-phase, while both $21 \mathrm{Cr} 11 \mathrm{Ni}$ form few $\sigma$-phase or they do not form it at all.
\end{abstract}

Keywords: Refractory austenitic stainless steels. Oxidation in air. High temperatures. $\sigma$ - phase.

\section{INTRODUCCIÓN}

Los aceros inoxidables para usos refractarios basan su buena resistencia a la oxidación a altas temperaturas en la formación de capas de óxidos que funcionan como barreras protectoras, reduciendo la velocidad de la reacción. En las condiciones reales de uso, además de la oxidación, pueden formarse fases fragilizantes que comprometen el comportamiento del acero. Este trabajo aporta algunos resultados en relación con estas cuestiones.

(*) ACERINOX, S.A. Centro de Investigación y Ensayos. 11379-Palmones (Los Barrios) Cádiz (España).

(**) Dpto. de Ciencias de los Materiales e Ingeniería Metalúrgica. Fac. de Ciencias Químicas. Univ. Complutense de Madrid. 28040-Madrid (España).

\section{PARTE EXPERIMENTAL}

La tabla I presenta la composición química de los tres aceros. Se emplearon probetas de $50 \mathrm{~mm} \times$ $50 \mathrm{~mm}$ obtenidas mediante cizallado y mecanizado de bordes de chapas comerciales, cuyo estado era de laminadas, recocidas y decapadas. Sus dimensiones se determinaron con ayuda de un calibre y un micrómetro, calculando la superficie y pesando cada probeta con una balanza de una precisión de $0,0001 \mathrm{~g}$.

El tratamiento térmico de oxidación al aire a $1.323 \mathrm{~K}$ durante $2 \mathrm{~h}$, se hizo en horno de mufla, ensayando seis probetas para promediar resultados. Tras el ensayo, las probetas se sacaron del horno y se enfriaron en un crisol de porcelana tapado, determinándose la ganancia de masa. Los óxidos 
TABLA I.- Composición química (\% masa) de los aceros inoxidables

TABLE I.-Chemical composition (mass \%) of the stainless steels

\begin{tabular}{|c|c|c|c|c|c|c|c|c|c|}
\hline Materiales & $\mathrm{Cr}$ & $\mathrm{Ni}$ & $\mathrm{Si}$ & $\mathrm{C}$ & $\mathrm{Mn}$ & $\mathrm{P}$ & $\mathrm{N}$ & $\mathrm{Mo}$ & Ln's \\
\hline $25 \mathrm{Cr} 20 \mathrm{Ni}_{(\mathrm{AISI} 310 \mathrm{~S})}$ & 24,59 & 19,3 & 1,20 & 0,064 & 0,39 & 0,023 & 0,0247 & 0,19 & - \\
$21 \mathrm{Cr}_{11 \mathrm{Ni}}(1)$ & 20,91 & 10,9 & 1,47 & 0,087 & 0,48 & 0,020 & 0,1780 & 0,18 & 0,036 \\
$21 \mathrm{Cr}_{11} \mathrm{Ni}_{(2)}$ & 20,97 & 10,96 & 1,88 & 0,075 & 0,42 & 0,024 & 0,1367 & 0,10 & 0,010 \\
\hline
\end{tabular}

Nota.- $\mathrm{Ln}$ 's $=\Sigma \%(\mathrm{Ce}, \mathrm{La}, \mathrm{Nd}, \ldots)$.

formados se estudiaron con DRX (Siemens 5000), sobre las superficies mayores de las muestras, y con MEB - EDS (Hitachi 570 - Kevex 8005, det. $\mathrm{Si}(\mathrm{Li}))$, sobre secciones metalográficas obtenidas con protección previa de los óxidos, mediante vaporización de platino y deposición electrolítica de níquel, antes del corte transversal y los pulidos metalográficos (1).

Los ensayos de envejecimiento ( $400 \mathrm{~h}$ a 1.073 $\mathrm{K})$ se hicieron con el mismo tipo de probetas, el mismo horno y también al aire. Las observaciones y los microanálisis de las fases se hicieron con un MEB(EC) - EDS (Zeiss DSM 982 GEMINI, LINK ISIS 200 det. Ge).

\section{RESULTADOS Y DISCUSIÓN}

\subsection{Oxidación al aire: 2 h a $1.323 \mathrm{~K}$}

Los mejores resultados los presentó el $25 \mathrm{Cr} 20 \mathrm{Ni}$, con $0,28 \mathrm{mg} \mathrm{cm}^{-2}(1,8 \mu \mathrm{m}$ de espesor teórico de la capa de óxidos), seguido del $21 \mathrm{Cr} 11 \mathrm{Ni}_{(1)}$ con $0,32 \mathrm{mg} \mathrm{cm}^{-2}(2,0 \mu \mathrm{m})$, y el $21 \mathrm{Cr} 11 \mathrm{Ni}_{(2)}$ con $0,47 \mathrm{mg} \mathrm{cm}^{-2}(3,0 \mu \mathrm{m})$. En los tres materiales, mediante DRX, se detectan señales correspondientes a óxidos de tipo $\mathrm{Cr}_{2-\mathrm{x}} \mathrm{Fe}_{\mathrm{x}} \mathrm{O}_{3}$ y a espínelas del tipo $\left.\mathrm{Mn}_{\left[\mathrm{Cr}_{2-\mathrm{x}}\right.}(\mathrm{Mn}, \mathrm{Fe})_{\mathrm{x}}\right] \mathrm{O}_{4}$.

A partir de las secciones metalográficas, se observa, también en todos los casos, que las capas de óxidos están bien adheridas a los materiales base y sus espesores reales, comprendidos entre 1 y 3 $\mu \mathrm{m}$, están en concordancia con los calculados a partir de los datos de termogravimetría (2). La figura 1 muestra una imagen de electrones retrodispersados y mapas de rayos $\mathrm{X}$ de silicio, manganeso, cromo $\mathrm{y}$ hierro de la misma área, correspondiente al material $25 \mathrm{Cr} 20 \mathrm{Ni}$. Se ven formas claramente poligonales en la parte externa de la capa de óxidos, especialmente ricas en manganeso y cromo; en la capa situada debajo de las anteriores, el cromo es prácticamente el único metal presente. El mapa de silicio muestra una precipitación interna perteneciente a fronteras de grano. La distribución de los elementos manganeso y cromo es coherente con los datos de DRX, pudiendo deducirse la presencia de espinelas ricas en $\mathrm{Cr}-\mathrm{Mn}$ en la capa más externa y óxidos tipo $\mathrm{Cr}_{2} \mathrm{O}_{3}$ más al interior. La figura 2 muestra la imagen de electrones retrodispersados del material $21 \mathrm{Cr} 11 \mathrm{Ni}_{(1)} \mathrm{y}$, aunque no se ofrecen los mapas de rayos $\mathrm{X}$, la distribución de silicio, cromo, manganeso y hierro coincide con la del $25 \mathrm{Cr} 20 \mathrm{Ni}$. La principal diferencia estriba en la mayor densidad de precipitados internos ricos en $\mathrm{SiO}_{2}$ en el $21 \mathrm{Cr} 11 \mathrm{Ni}_{(1)}$. Exactamente los mismos comentarios son aplicables al $21 \mathrm{Cr}_{11 \mathrm{Ni}}(2)$ (Fig. 3).

\subsection{Envejecimiento: 400 h a $1.073 \mathrm{~K}$}

En el $21 \mathrm{Cr} 11 \mathrm{Ni}_{(1)}$ no se forma fase $\sigma$, siendo escasa -alrededor del $1 \%$ en vol.- en el $21 \mathrm{Cr} 11 \mathrm{Ni}_{(2)}$ y relativamente abundante $-10 \%$ en vol.- en el 25Cr20Ni. De los microanálisis, se pueden deducir las fórmulas siguientes:

$$
\begin{aligned}
& \sigma_{25 \mathrm{Cr} 20 \mathrm{Ni}_{(1)}}: \mathrm{Cr}_{0,87} \mathrm{Fe}_{0,91} \mathrm{Ni}_{0,17} \mathrm{Si}_{0,04} \mathrm{Mo}_{0,01} \\
& \sigma_{21 \mathrm{Cr} 11 \mathrm{Ni}(2)}: \mathrm{Cr}_{0,76} \mathrm{Fe}_{1,081} \mathrm{Ni}_{0,10} \mathrm{Si}_{0,06}
\end{aligned}
$$

Se puede establecer una clara proporcionalidad composicional entre las aleaciones originales y las fases $\sigma$ formadas, de modo que la fase $\sigma$, evolucionada a partir del $25 \mathrm{Cr} 20 \mathrm{Ni}$, es más rica en cromo y níquel que la del $21 \mathrm{Cr} 11 \mathrm{Ni}_{(2)}$.

También, y a partir de las composiciones químicas de los tres aceros, se calculan los niveles medios de energía de los orbitales $\mathrm{d}(\overline{M d})$ y $\operatorname{los} \mathrm{Md}_{\text {Crit }}$ (a $1.073 \mathrm{~K}$ ), deduciéndose la tendencia de cada uno de los tres aceros a la formación de fase $\sigma$ (3). Según este cálculo, la mayor tendencia (un $4 \%$ en vol.) corresponde al $25 \mathrm{Cr} 20 \mathrm{Ni}$, mientras que resulta ser sólo del $1,5 \%$ en los dos aceros $21 \mathrm{Cr} 11 \mathrm{Ni}$, lo que globalmente coincide con lo observado experimentalmente, apuntándose que las diferencias extremas encontradas en el $25 \mathrm{Cr} 20 \mathrm{Ni}(10 \%$ en vol.) y $21 \mathrm{Cr} 11 \mathrm{Ni}_{(1)}(0 \%)$ se apoyan en los niveles de cromo del primero y los niveles altos de $\mathrm{N}(0,18$ $\%)$ en el segundo. 

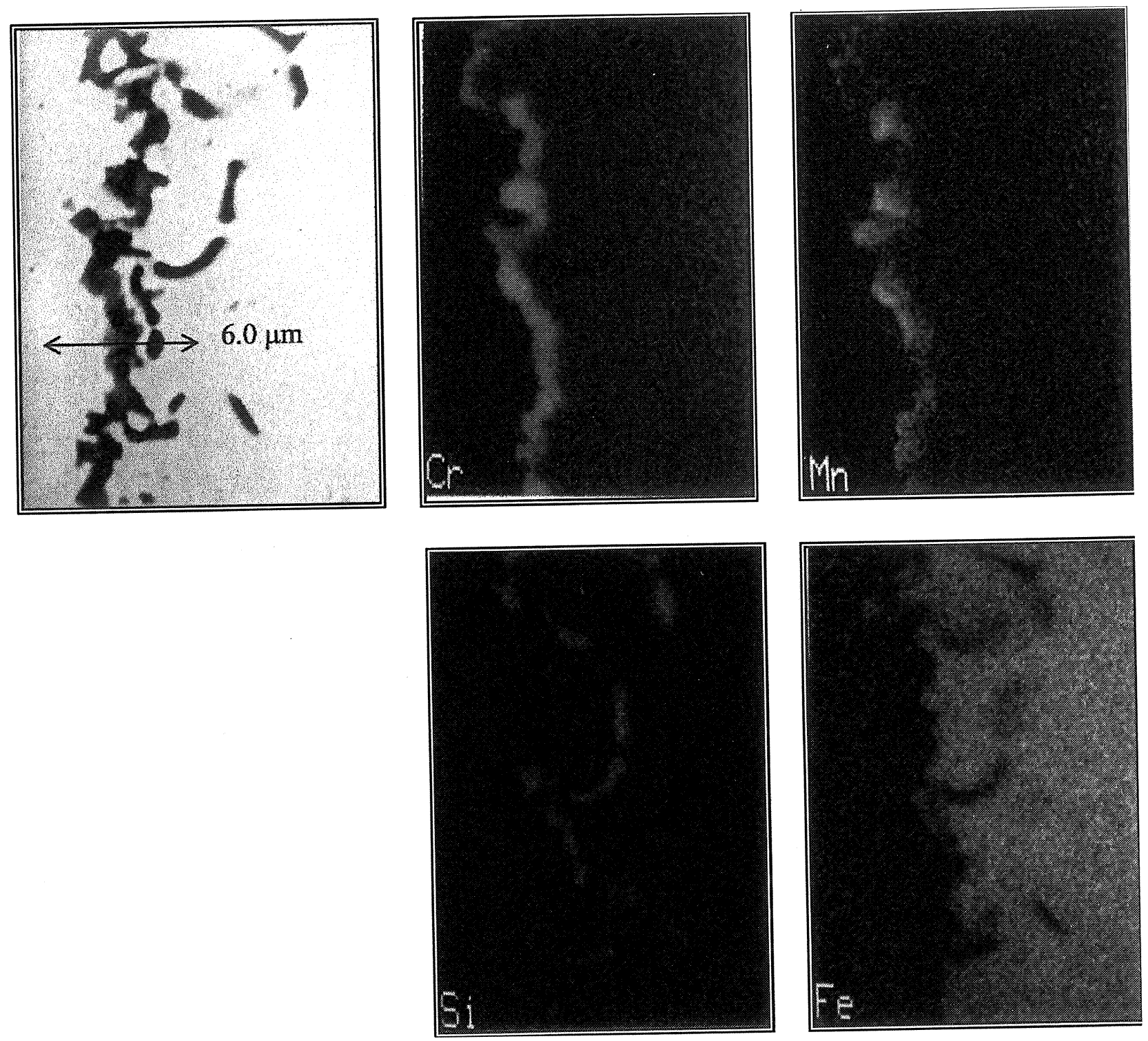

FIG. 1.-25Cr20Ni : 2h $1323 \mathrm{~K}$

Imagen de electrones retrodispersos y mapas de rayos-X

FIG. 1.-Backscattered electron images and X-ray mappings

\section{CONCLUSIONES}

Del estudio de la oxidación al aire (2 h a 1.323 $\mathrm{K})$, se deduce que los tres materiales tienen un adecuado comportamiento refractario, algo mejor, cuantitativamente, el $25 \mathrm{Cr} 20 \mathrm{Ni}$, seguido muy de cerca por el $21 \mathrm{Cr} 11 \mathrm{Ni}_{(1)}$ con $(0,04 \%$ de lantánidos $)$ y luego el $21 \mathrm{Cr}_{11} \mathrm{Ni}_{(2)}$ (con 0,01 de lantánidos). Del estudio mediante DRX y MEB-EDX se deduce que los tres aceros basan su buen comportamiento en la formación de capas de $\mathrm{Cr}_{2} \mathrm{O}_{3}$ bajo las que existen precipitados ricos en $\mathrm{SiO}_{2}$, tanto en la barrera óxido-metal, como en precipitados internos, más abundantes estos últimos en los materiales
$21 \mathrm{Cr} 11 \mathrm{Ni}$. Por encima de las capas de $\mathrm{Cr}_{2} \mathrm{O}_{3}$, ya en la interfase atmósfera-óxido, crecen cristales de $\mathrm{MnCr}_{2} \mathrm{O}_{4}$.

$\mathrm{El}$ envejecimiento térmico $(400 \mathrm{~h}$ a $1.073 \mathrm{~K})$ provoca la formación de un $10 \%$ en vol. de fase $\sigma$ en el $25 \mathrm{Cr} 20 \mathrm{Ni}$, mientras que sólo se forma un $1 \%$ en el $21 \mathrm{Cr}_{11} \mathrm{Ni}_{(2)}$ y nada en el material $21 \mathrm{Cr} 11 \mathrm{Ni}_{(1)}$, en buena aproximación a los cálculos teóricos de las tendencias formadoras de fase $\delta$ (niveles de energía de los orbitales $d$ ). Los microanálisis EDX de las fases ponen en evidencia composiciones químicas proporcionales a las de las aleaciones originales. En la inhibición de la formación de fase $\sigma$ en el $21 \mathrm{Cr} 11 \mathrm{Ni}_{(1)}$ actuaría fundamentalmente la mayor aleación de nitrógeno. 


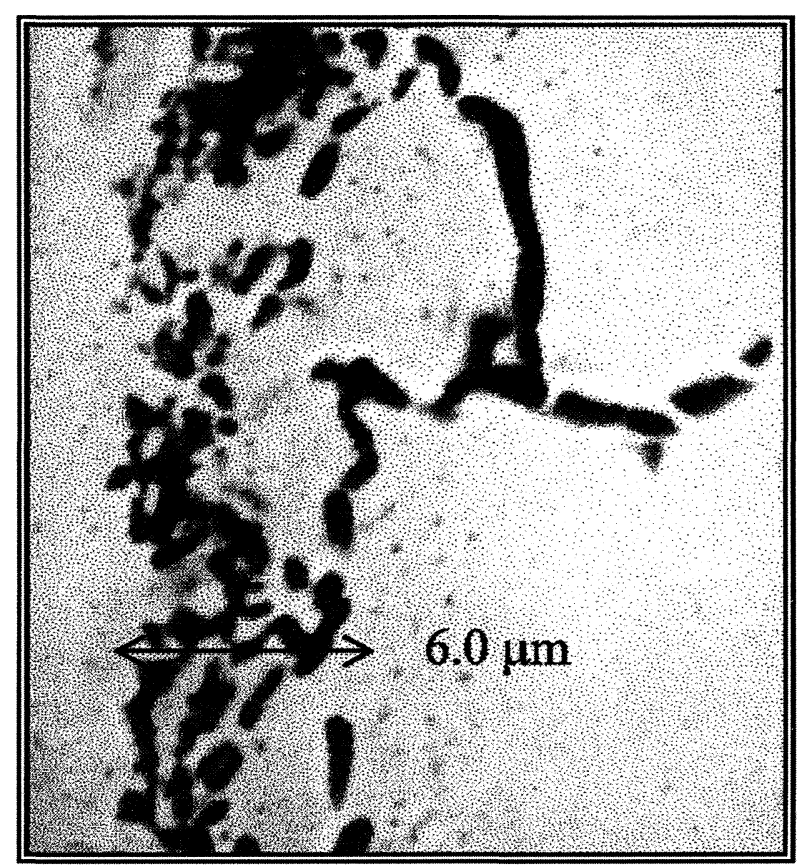

FIG. 2.- $21 \mathrm{Cr} 11 \mathrm{Ni}{ }_{(1)}: 2 \mathrm{~h} 1323 \mathrm{~K}$ Imagen de electrones retrodispersos

FIG. 2.-Backscattered electron images

\section{REFERENCIAS}

(1) Botella, J. Tesis Doctoral. Universidad Complutense de Madrid. 1996.

(2) Caplan, D. y Cohen, M. Corrosion NACE. 15, 1958: 141t.

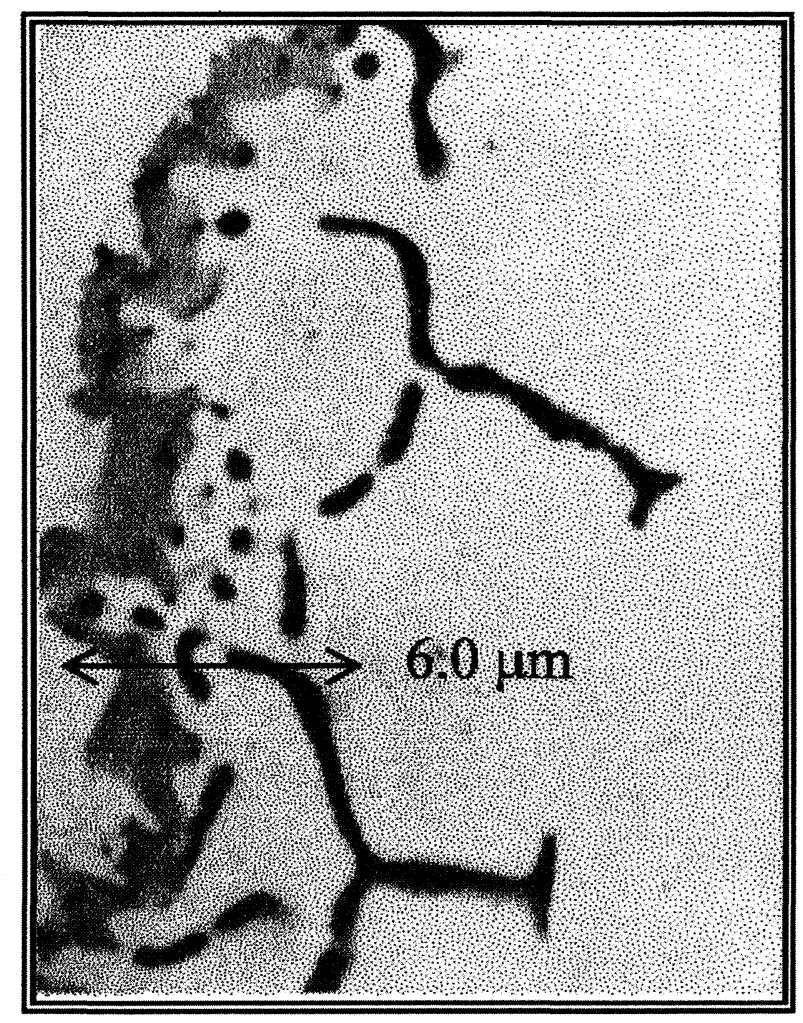

FIG. 3.- $21 \mathrm{Cr} 11 \mathrm{Ni}_{(2)}: 2 \mathrm{~h} 1323 \mathrm{~K}$ Imagen de electrones retrodispersos

FIG. 3.-Backscattered electron images

(3) Uggowitzer, P.J., Magdowski, R. y Speidel, M.O. Innov. Stainless Steel Conf. Florencia (Italia) 1993: 2.359-2.372. 